\title{
AU CROISEMENT DE L'HISTOIRE ET DE LA LITTÉRATURE : ANALYSE DU RÉCIT DE TÉMOIGNAGE À LA SUITE DU GOUVERNEMENT SERBE, DE NICH À CORFOU, 20 OCTOBRE 1915-19 JANVIER 1916 D'AUGUSTE BOPPE (1917)
}

Les relations franco-serbes s'inscrivent dans une longue tradition qui remonte au Moyen-Âge. Elles s'étaient intensifiées au cours du XIX ${ }^{\mathrm{e}}$ siècle et renforcées par la « fraternité d'armes » durant la Grande Guerre (1914-1918). De nombreux documents et souvenirs personnels témoignent de cette réalité. Notre étude consiste donc en l'analyse d'extraits du témoignage $\grave{A}$ la suite du gouvernement Serbe, de Nich à Corfou, 20 octobre 1915 - 19 janvier 1916 (1917) d'Auguste Boppe, diplomate et écrivain français. Certaines perspectives orienteront notre recherche : au vu du fait que la littérature de témoignage - genre littéraire qui conjugue les domaines de la littérature et de l'histoire - occupe un statut tout particulier dans la pensée littéraire contemporaine en France, nous nous interrogerons sur les enjeux littéraires, historiques et éthiques de cet « acte de témoignage ». L'intérêt de l'analyse est donc double ; éthique et historique d'abord, il s'agit de dire la vérité du vécu au nom des morts, et littéraire et esthétique par la suite, de rendre compte de l'expérience de guerre en passant inévitablement par le prisme subjectif de la narration.

Mots-clés : genre littéraire, témoignage, histoire, Grande Guerre, relations franco-serbes

L'année 2019 est emblématique, et pour plusieurs raisons : avant tout, le monde entier célèbre les deux grands événements historiques : l'armistice signé en 1918 et le traité de Versailles signé en 1919 qui marquent la fin de la « Grande guerre » - la guerre pendant laquelle les deux peuples, français et serbe, ont fait preuve d'une non moins grande « fraternité d'armes ». C'est

\footnotetext{
${ }^{1}$ dragana.cvetkovic@univ-lorraine.fr

${ }^{2}$ Réseau UFA - Université franco-allemande
} 
la période durant laquelle les deux pays alliés ont entretenu des relations étroites (tout d'abord politiques et économiques, mais aussi culturelles et intellectuelles). L'année 2019 est également l'occasion de saluer les 180 ans du rétablissement des relations franco-serbes, «Les 180 ans... d'une délicate attention $^{3} »$.

Les relations franco-serbes ne se réduisent pas uniquement à ces 180 ans de relations institutionnelles. En réalité, les contacts franco-serbes remontent au Moyen Âge avec le mariage d'Hélène d'Anjou avec le roi Uroš I Nemanjić en 1245. Bien qu'il se soit fait pour des raisons d'alliance familiale (qui sousentend, évidemment, l'alliance politique et militaire), celui-ci a marqué le début d'une longue tradition diplomatique. Néanmoins, c'est n'est qu'au mois de juillet 1839 que « l'Agence consulaire française de Belgrade est devenue un consulat ${ }^{4} »$, dix ans après l'acquisition de l'autonomie serbe. ${ }^{5}$

Les rencontres littéraires, culturelles, politiques, historiques étaient nombreuses tout au long du XIX ${ }^{\mathrm{e}}$ siècle et renforcées par la « fraternité d'armes » au cours de la Grande Guerre (1914-1918). C'est notamment à la suite de ce conflit qu'est inauguré dans le parc de Kalemegdan à Belgrade un monument qui symbolise les relations franco-serbes. En mémoire de la reconnaissance à la France, il porte l'inscription suivante: «Nous aimons la France comme elle nous a aimés ». Ce monument, construit par le sculpteur Meštrović, représente l'incarnation sculpturale de la mémoire de guerre et témoigne des liens solides qui ont uni les deux pays entre 1914 et 1918. Pourtant, pour accéder à la parole vive sur cette triste réalité, le recours à un autre moyen artistique est nécessaire - c'est le témoignage de la guerre.

Stanislav Sretenović rappelle que la construction de ce monument, selon le contexte historique dans lequel il a paru, avait certes une visée diplomatique et politique :

« L'inauguration du monument à la France le 11 novembre 1930 à Belgrade ne représente pas seulement un événement symbolique qui évoquait l'aide matérielle et morale de la France à la Serbie pendant la Grande Guerre. Elle signifie la volonté politique et diplomatique de la France d'agir par rapport à la situation intérieure et extérieure du royaume de Yougoslavie où le régime personnel du roi Alexandre Karadjordjević venait d'être établi» (SRETENOVIĆ $2012: 31)$.

\footnotetext{
${ }^{3}$ Nous reprenons le titre de la présentation consacrée à ce sujet sur le site de l'Ambassade de France en Serbie : Les 180 ans... d'une délicate attention, source https://rs.ambafrance.org/ Les-180-ans-d-une-delicate-attention, dernière consultation le 3/11/2019.

${ }^{4}$ Selon les archives de 1'Ambassade de France en Serbie : https://rs.ambafrance.org/Juillet1839-1-Agence-consulaire-francaise-de-Belgrade-devenait-un-consulat, dernière consultation le 03/11/2019.

${ }^{5}$ L'autonomie réelle de la Serbie est atteinte en 1829 par le Traité d'Andrinoples, la monarchie serbe n'étant reconnue par les Ottomans qu'en 1830.
} 
Or, pour comprendre cette « volonté politique et diplomatique française» liée au projet de la création de l'État yougoslave, il faut revenir en 1914 : l'année durant laquelle Auguste Boppe, diplomate français, envoyé « en qualité de Ministre de France en Serbie » prend connaissance du projet territorial yougoslave. L'ambition des Slaves du Sud selon Pavlović ${ }^{6}$ consiste en "l'incorporation des régions occidentales et septentrionales de l'espace yougoslave allant du Banat à l'Est jusqu'à la Carinthie à l'Ouest dans le futur état des Slaves du Sud » (PAVLOVIĆ 2006 : 171). Nombreux sont les historiens qui adhèrent à la thèse selon laquelle la France détient la responsabilité de la création du Royaume des Serbes, Croates et Slovènes (Royaume SHS). Cette influence diplomatique est à la fois externe (les questions de la délimitation des frontières) et interne (la problématique de l'organisation interne du Royaume).

En tenant compte des événements historiques des années 1914-1918 et de l'importance de la mission diplomatique française en Serbie durant cette période, nous pouvons affirmer que le mandat d'Auguste Boppe a bien orienté le cours de l'histoire yougoslave. En outre, l'activité de Boppe ne s'est pas uniquement réduite aux missions d'ordre politique ; il a été (et peut être avant tout) « un homme de lettres ». Écrivain et diplomate, sa double position lui a conféré une légitimité particulière. «En poste à Belgrade à titre d'attaché, en 1891, puis à Cetinje (chargé d'affaires), en 1901-1902, c'est en qualité de Ministre de France en Serbie pendant la Première Guerre mondiale, qu'il fit la retraite avec l'armée serbe, en 1915-1916, » selon la note biographique de Mihailo Pavlovićc ${ }^{7}$. De cette traversée, il fait paraître le récit intitulé À la suite du gouvernement Serbe, de Nich à Corfou, 20 octobre 1915-19 janvier 1916, ${ }^{89}$ publié dans les numéros du 15 décembre 1916 et du 1er janvier 1917 de la Revue des Deux Mondes, réédité aux éditions Bossard ${ }^{10}$ (1917). Notre objectif est d'analyser les extraits de ce récit

\footnotetext{
${ }^{6}$ À propos des questions relatives au rôle de la France et le programme yougoslave voir : PAVLOVIĆ, Vojislav. " La France et le programme yougoslave du gouvernement serbe. » Balcanica, vol. 37, (2006) : 171-193.

${ }^{7}$ La note de Mihailo Pavlović sur Auguste Boppe et les extraits de l'ouvrage À la suite du gouvernement Serbe, de Nich à Corfou, 20 octobre 1915-19 janvier 1916 sont reproduits dans la revue électronique Serbica d'après : Témoignages français sur les Serbes et la Serbie 19121918, choix de textes, notes de présentation, traduction et commentaires par Mihailo Pavlović, (Francuzi o Srbima i Srbiji : 1912-1918, izbor, uvodni tekstovi, prevod i komentari Mihailo Pavlović), édition bilingue, Belgrade : Narodna knjiga, 1988, p. 156-165.

${ }^{8}$ BOPPE, Auguste. À la suite du gouvernement Serbe, de Nich à Corfou, 20 octobre 1915-19 janvier 1916. Paris : Éditions Bossard, 1917.

${ }^{9}$ Nous renvoyons à l'édition récente de cet ouvrage traduit en serbe : ĐOKIĆ, Dušan, VITEZOVIĆ, Milan. Za srpskom vladom od Niša do Krfa: od 20. oktobra 1915. do 19. januara 1916. Beograd : Čigoja štampa, 2014. [orig.] ЂОКИЋ, Душан, ВИТЕЗОВИЋ, Милан. $3 a$ српском владом од Ниша до Крфа: од 20. октобра 1915. до 19. јануара 1916. Београд: Чигоја штампа, 2014.

${ }^{10}$ Les éditions Bossard, créées dans les années 1910 ont beaucoup publié sur la guerre de 1914 -
} 
parus dans le dossier spécial La Serbie dans la Grande Guerre 1914-1918 (2014) de la revue électronique Serbica ${ }^{11}$ tout en se focalisant sur la poétique du genre testimonial et en adoptant une perspective historienne et littéraire.

Le rôle de Boppe, répétons-le, est donc double : il est à la fois acteur et spectateur des mutations historiques dont il rend compte dans son témoignage. C'est le clivage de sa position - à la fois active et passive - qui est à l'origine de l'œuvre de Boppe. Or, cette fracture primaire entre les nécessités fondatrices, la factualité de l'histoire et l'esthétique de la littérature, l'objectivité d'historien et la subjectivité d'écrivain, est aux sources du témoignage en tant que genre littéraire dont il nous semble pertinent de rappeler les prémisses historiques et théoriques.

Le témoignage de guerre peut-il être considéré comme un genre littéraire ? Si la question, de manière systématique, a été posée pour la première fois par Norton $\mathrm{Cru}^{12}$ qui a survécu à la Grande Guerre, et si l'histoire de ce genre littéraire a été retracée par Jean-Louis Jeannelle dans son essai Pour une histoire du genre testimonial (2004) et étudié par Charlotte Lacoste dans sa thèse Le témoignage comme genre littéraire en France de 1914 à nos jours ${ }^{13}$ soutenue en 2011 à l'Université Paris 10, l'intérêt pour ce " genre à part » ne cesse de prendre de l'ampleur.

Quel statut peut-on accorder au témoin - dont la parole est crédible, qui atteste ou conteste les grands récits historiques ? Selon François Hartog, le témoin est " une figure centrale des communautés humaines. Par son entremise, on peut relier le passé et le présent : le passé de ce qui a eu lieu et le présent de son attestation » (HARTOG 2017 : 169). Si la notion au premier abord renvoie à l'épistémologie des sciences juridiques et théologiques, elle n'est pas moins importante dans la théorie littéraire. Le caractère éthique de l'attestation répond à la déontologie de chacune de ces trois disciplines : le témoin garantit la vérité de ce qu'il atteste. Selon le code pénal français, l'acte de parole du témoin doit être conforme aux règlements juridiques et doit garantir l'exactitude des faits rapportés. D'ailleurs, les religions monothéistes reposent sur le témoignage : le judaïsme, l'islam et encore, le christianisme

1918 et sur les conséquences de ce conflit ; cette maison d'édition a traité la problématique de nationalité en Europe de l'Est et dans les Balkans.

${ }^{11}$ La revue en ligne Serbica est un projet conjoint de l'université Bordeaux Montaigne, EA 4593 CLARE, Ministère de la culture de la République de Serbie et Maison des Sciences de l'Homme d'Aquitaine. Elle est dirigée par Milivoj Srebro. https://serbica.u-bordeaux-montaigne.fr/index. php/presentation

${ }^{12}$ CRU, Norton. Témoins : essai d'analyse et de critique des souvenirs de combattants édités en français de 1915 à 1928, Paris : Les Étincelles, 1929 ; version rééditée et abrégée $D u$ Témoignage, coll. «Les documents bleus », Paris : Gallimard, 1930.

${ }^{13}$ Le témoignage comme genre littéraire en France de 1914 à nos jours, sous la direction de Tiphaine Samoyault et de François Rastier. 
accordent une place primordiale aux témoins qui garantissent la transmission de la parole divine. Or, la tradition testimoniale remonte encore plus loin. Dans l'Antiquité, les témoins « grecs pouvaient jurer en faisant appel à Zeus, selon la formule Istô Zeus (« Que Zeus l'atteste », « Que Zeus soit témoin ») ; les Romains, quant à eux, disaient plutôt Audi Jupiter (" Entends, Jupiter »). En grec, l'étymologie du mot « témoin », martus, conduit vers le radical d'un verbe signifiant « se souvenir » (smarati en sanscrit ; en grec merimna, qui a donné en latin memor[ia]). Le témoin est mémoire » (HARTOG $2007: 248$ ).

$\mathrm{Si}$, selon Jeannelle (2004: 87) « la parole humaine reste relativement fragile à l'encontre des systèmes idéologiques $\|^{14}$, alors l'émergence d'un tel genre littéraire s'inscrit dans une double nécessité dialectique : singulière et subjective d'une part - rapporter la vérité de l'expérience personnelle au front; et plurielle et objective d'autre part - donner accès à la possible identification pour tous ceux qui étaient présents aux événements traumatisants et pourront confirmer le récit fait à titre individuel en disant : « c'était ainsi ». Norton Cru insiste sur le passage de la représentation d'une bataille « imaginée et imaginaire » à la représentation réelle, c'est-à-dire à la transmission de l'expérience vécue par les combattants. Selon cet auteur, les intellectuels, nourris de légendes et de mythes et n'ayant aucune expérience au front, donnent une fausse et dangereuse image de la guerre : "Les grands chefs ne pouvaient pas savoir car leur intelligence seule était en contact avec la guerre, et la guerre ne se laisse pas concevoir par l'intelligence seule » (CRU 2002 : 9). La légitimité de témoin est donc proportionnelle au degré d'implication au front.

Factualité ou émotion? Force est de constater que le récit de témoignage transgresse cette franche dichotomie. De plus, les témoignages de guerre et de catastrophes historiques passent au travers du prisme de la narration et de la remémoration strictement personnelle, d'où les qualités de littéralité de ces récits. Les enjeux du témoignage en tant que genre littéraire selon Jeanelle (2004 : 83) sont triples. Tout d'abord, l'enjeu est d'ordre « éthique »-dire la vérité, rien que la vérité du vécu et de l'expérience au nom des morts. L'enjeu est également « méthodologique », autrement dit, historico-politique - dire la vérité du vécu contre les grands récits historiques et idéologiques pour conserver la mémoire de la réalité des grands événements. Enfin, l’enjeu est « esthétique »-cette mémoire passe inévitablement par le biais de la narration ; elle est subjective, guidée par les émotions.

Commençons par la factualité : Jeanelle insiste sur le caractère factuel et neutre du témoignage, sur l'obligation «éthique » de témoigner de l'expérience vécue en mémoire des victimes. D'ailleurs selon cet auteur : « Le témoin ne jouit d'aucun surplomb ; il se tient aux lieux les plus risqués de l'Histoire : ses

${ }^{14}$ La question a été également abordée par Paul Ricœur dans son œuvre La mémoire, l'histoire, l'oubli (2000). 
angles, ses points morts ou ses friches. » (2004: 89). La pratique testimoniale de Boppe correspond à cette exigence théorique. En allant vers Scoutari afin d'effectuer l'embarquement à Saint-Jean de Médua, Boppe est confronté à des paysages terrifiants : les soldats, les réfugiés, les chevaux sur le point de mourir se trouvent devant lui. Néanmoins, la vision de Boppe est neutre, le ton demeure calme ; aucune surcharge émotive, aucun recours au pathétique ne sont perceptibles. La description suivante de l'armée serbe le confirme :

" Ceux qui nous ont suivis sur cette route d'épouvante ont vu des hommes mourir. Cette tristesse nous a été épargnée, mais bien des vivants ne valaient guère mieux que des morts parmi les soldats des cantonnements d'Alessio »(BOPPE 1917 : 128).

Cependant, un double mouvement régit la position de Boppe, c'est à la fois le sentiment de l'identification et de l'aliénation à l'égard des soldats serbes. Soucieux de la destinée de ceux qui partagent l'expérience de cet espace-temps critique, l'auteur essaie de se mettre dans la position des soldats :

« Mais voici qu'aux convois se mêlent des soldats ; ils marchent, ils marchent en file ininterrompue ; ils ont la tête basse, la mine farouche ; leurs officiers en nous croisant nous regardent d'un air sombre ; que se passe-t-il dans ces cerveaux ?» (BOPPE 1917 : 131).

Les signes de ce mouvement dialectique (solidarité-aliénation) sont de l'ordre du non-dictum, ils ne sont que suggérés : « que se passe-t-il dans ces cerveaux ? » pourrions-nous interpréter cela comme « quelle attitude prendre devant la gravité de la situation ? » en prenant en compte les conditions existentielles des soldats et des réfugiés serbes. Pourtant, le sentiment d'aliénation est saisi dans le regard des officiers : " leurs officiers en nous croisant nous regardent d'un air sombre », signe d'une nette différenciation entre le statut privilégié du corps diplomatique dont les agents seront les premiers à s'embarquer sur le bateau si attendu et l'armée et les réfugiés serbes « vieillards, femmes et enfants, qui depuis des semaines attendaient, sans abri, un bateau chaque jour promis ».

L'auteur est donc conscient du fait qu'il retrace l'histoire de ceux qui vont mourir. Il faut noter que le terme histoire est employé dans sa double signification : Histoire en tant qu'étude du passé et histoire en tant que récit littéraire. Ces deux sens se complètent dans le récit. À la fois littéraire et documentaire, le texte de Boppe permet donc de reconstituer la totalité de la traversée héroïque. Or, quelle méthodologie faut-il adopter pour aboutir à une telle reconstitution?

L'enjeu " méthodologique », deuxième des trois enjeux fondateurs sur lesquels repose le récit testimonial selon Jeanelle, « touche aux modes d'établissement de la vérité et concerne en priorité le juge et l'historien, tous 
deux confrontés, au cours de leur travail de reconstitution des faits passés. » (2004 : 83) En retraçant au jour le jour l'histoire de la traversée de l'armée serbe (du 20 octobre 1915 au 19 janvier 1916), Boppe associe les savoirs historiques et géographiques. Dans cette perspective, la datation des passages accompagnée par l'évocation des toponymes, (à titre d'exemple « Nich, Ipek, Prizren, Diakovo, Detchan, Scutari, Saint-Jean de Médua, Corfou ») démontre le caractère informatif de son témoignage. La valeur documentaire de ce récit est d'autant plus accentuée que la précision méthodologique de Boppe évolue - l'auteur ne se contente pas de noter les dates, il prend acte de chaque modification, en évoquant l'heure et la durée des activités :

« Le 14 janvier, dès trois heures du matin, la caravane se préparait sous la surveillance de M. Briot, notre hôte dévoué ; à six heures, tout était prêt ; la caravane du ministre d'Angleterre passait devant notre porte ; nous la suivons »(BOPPE 1917 : 126).

Le changement de mode d'énonciation est révélateur : l'emploi de l'imparfait ( « la caravane se préparait », « tout était prêt », " la caravane [...] passait ») fait écho à l'écriture historienne - l'auteur s'adresse ainsi à la postérité en prenant ses distances à l'égard des actions qui viennent de se produire. Subitement, et peut-être inconsciemment, la position de l'auteur change ; par l'emploi du présent, il se réapproprie la position du témoin qui saisit « sur le vif» les changements de la situation : «nous la suivons ».

La lecture du témoignage de Boppe donne accès à plusieurs aspects de l'information géographique dont nous proposons la qualification suivante :

a) la description et la configuration du terrain : " La plaine entre la Bojana et le Drin est monotone »; « la route est sèche, facile; elle est très animée ; des groupes, des cavaliers isolés, des piétons en grand nombre se hâtent vers Alessio et Saint-Jean de Médua ». (BOPPE 1917 : 127) Chaque modification est précisément notée : «À partir de Kakarich, la route devient mauvaise ; des fondrières ralentissent notre marche, et ce n'est que tard dans l'après-midi que nous apercevons de l'autre côté du Drin la forteresse d'Alessio. »;

b) la description des conditions climatiques : «à l'approche de la mer nous sentions le vent se lever, bientôt il souffla en tempête ; nous traversions un véritable grain de neige fondue, de pluie glaciale, sous cette rafale les chevaux hésitaient » (BOPPE 1917 : 132);

c) la description démographique et éthologique sur laquelle nous portons une attention particulière.

Force est de constater que le récit de Boppe est doublement historique. Son texte ne représente pas seulement une source d'information pour la postériorité sur la grande traversée durant la Première Guerre mondiale, il est 
aussi complété par les données historiques et ethnologiques sur les régions que le témoin traverse. En passant par Ipek (Peć), l'auteur informe ses lecteurs de l'histoire du patriarcat d'Ipek (Pećka patrijaršija) et étend sa réflexion à la grande traversée « de l'Ipek à Karlowitz menée par le patriarche Arsène Tchernojevitch » (en 1690). Boppe met en avant le caractère répétitif de l'Histoire grâce à ce toponyme géographique :

« Autour du patriarcat de Karlowitz, héritier du patriarcat d'Ipek, les Serbes purent longtemps se développer, mais le joug de l'Autrichien leur est devenu aussi odieux que celui du Turc et, par un singulier retour de l'histoire, lpek voit maintenant revenir, fuyant devant les Austro-Allemands, les descendants de ceux que les Turcs ont chassés » (BOPPE 1917 : 74).

L'auteur ne prend pas seulement acte de l'expérience immédiate à laquelle il est confronté, il met en lumière les enjeux historiques, éthologiques et géopolitiques pour contextualiser l'espace-temps dont il rend compte. En évoquant le passage d'Ipek, Boppe a recours aux réalités historiques incontournables pour comprendre et situer le présent :

« Les musulmans et les Albanais ont jusqu'ici respecté l'antique demeure patriarcale, ses églises, ses trésors ; les envahisseurs autrichiens et bulgares auront-ils la même tolérance ? Ne voudront-ils pas faire disparaître ces monuments du serbisme ? Ne chercheront-ils pas à rendre les Albanais complices de leurs crimes? » (BOPPE 1917 : 75)

L'auteur met au clair les raisons historiques de l'inquiétude de l'archevêque d'Ipek et de son peuple. Or, l'inquiétude est le sentiment qui domine le récit. À travers elle, l'auteur expose la tragédie humaine de la traversée et de ses conséquences. On peut ainsi affirmer que le récit de témoignage n'est pas réductible à sa visée strictement informative et référentielle : la lecture des textes testimoniaux donne accès à la dimension affective. Par l'entremise de la portée esthétique, le genre testimonial transgresse le domaine du factuel et du référentiel - autrement dit le domaine de l'historique, ce qui justifie de qualifier les témoignages de littéraires.

L'enjeu esthétique est le troisième pilier fondateur du genre testimonial selon Jeanelle (2004 : 83). Il identifie la problématique suivante à ce sujet : « mais dans le récit en prose lui-même se pose de manière particulièrement présente la question de la conciliation entre visée référentielle et ambitions esthétiques. \Comment concilier cette dialectique ? Le prochain volet de notre réflexion se réfère au questionnement du théoricien « quels moyens poétiques sont mis au service du témoignage ? (JEANELLE $2004: 88$ )

La dynamique du récit révèle le caractère poétique du texte de Boppe en suivant le rythme de passage de l'armée (strate factuelle), l'auteur met en scène la dynamique intérieure (strate affective) qu'il associe à la représentation 
des paysages. Ainsi, le texte se construit en tant qu'espace mobile où s'opère le « partage du sensible ${ }^{15}$ » entre l'auteur, les personnes présentes - soldats, officiers, réfugiés, représentants du gouvernement serbe et les lecteurs. Ce « partage » ne se saisit que dans le non-dictum et dans la suggestion : l'esthétique de l'inquiétude et de l'angoisse trouve paradoxalement son expression dans le silence. Les contrastes, la gradation, la mise en attente, la modulation des plans de la représentation, les différentes longueurs de séquences textuelles sont les exemples des moyens poétiques dont Boppe fait usage avec virtuosité pour traduire le sentiment ambiant d'angoisse.

Analysons à cet égard la description du départ de ScutariL'Embarquement à Saint-Jean de Médua. Le contraste entre les dynamiques qui animent la représentation des plans des séquences consécutives (la motion - le calme - la motion) contribue à la construction d'une vision vibrante. Pour reprendre les termes musicaux, vibrato et, surtout, decrescendo, au vu de la réduction progressive de la longueur séquentielle, sont deux modalités structurelles qui donnent forme au récit :

« La route est sèche, facile ; elle est très animée ; des groupes, des cavaliers isolés, des piétons en grand nombre se hâtent vers Alessio et Saint-Jean de Médua. Des détachements de cavalerie dirigés sur Durazzo nous dépassent.

La plaine entre la Bojana et le Drin est monotone ; le ciel est gris ; les villages, rares, paraissent abandonnés ; une impression générale de tristesse plane sur la région.

Des vols de corbeaux tachent de noir l'horizon » (BOPPE 1917 : 127).

La modulation de la position visuelle, en allant du macro au micro plan met en avant l'effet de dynamisation. La gradation et l'attention portée aux détails sont constamment mises en exergue. Dans cette optique, Boppe évoque « les cadavres de chevaux » dont la route « en est comme pavée », ce qui rend le passage difficile à supporter. De cette vision globale, l'attention de l'auteur s'arrête à un détail singulier, au moment où « épuisé, le cheval d'un soldat ou d'un réfugié tombe ». L'homme décide dès lors d'abandonner l'animal et de continuer la traversée en portant la charge :

«Un instant le cheval reste couché, puis, comme dans un mouvement de lassitude et de désespoir, il se jette sur le côté et, quand on passe auprès de lui, on le voit la tête étendue sur le sol, l'œil éteint, la bouche haletante. Il meurt et, quelques mètres plus loin, un autre meurt comme lui » (BOPPE 1917 : 128).

La stratégie stylistique de la gradation et de la mise en attente dont la cible est dévoilée par la suite renforce la dramatisation : « Ceux qui nous ont suivis sur cette route d'épouvante ont vu des hommes mourir » (BOPPE 1917 : 128). Le lecteur se trouve face à des modifications consécutives qui suivent

${ }^{15}$ Nous reprenons le terme de l'esthétique de Jacques Rancière. Voir RANCIÈRE, Jacques. Le partage du sensible. Esthétique et politique. Paris : La Fabrique Editions, 2000. 
le mouvement du général vers le particulier et du particulier vers le général : chevaux-cheval-homme-hommes.

Or, l'effet dramatique est une constante stylistique du récit de Boppe. Dans cette perspective, il convient de comprendre en quoi la structure globale de la séquence XIV de ce témoignage « Départ de Scutari. L'Embarquement à Saint-Jean de Médua » correspond à la structure de la tragédie classique. L'auteur met en scène les jeux du clair-obscur - nous suivons l'intensification progressive de la lumière du jour naissant qui fait écho à la progression de l'action dramatique. Les étapes du modèle actantiel (exposition-nœuddénouement) peuvent être identifiées ; tout d'abord, l'exposition de la scène correspond à la montée de la lumière : " dans la lumière naissante, nous longeons la forteresse. » Par la suite, Boppe rencontre le médecin-major Blanc qui lui fait parvenir la nouvelle :

« Ce dont [il] est sûr, en tout cas, c'est du départ des 1200 soldats dont il vient de passer la visite ; il les a vus quitter Alessio et se mettre en route en chantant ; il pense qu'ils sont maintenant en train de monter sur le bateau qui les conduit vers le salut »(BOPPE 1917 : 129-130).

L'espoir, la promesse du salut, la venue du bateau si longuement attendu répondent à la vision claire, matinale, « chantante ». Néanmoins, ce nœud dramatique est suivi par les péripéties : le bateau Città-di-Bari n'est pas destiné aux soldats et réfugiés serbes, ceux qui seront pris à son bord, ce sont les représentants du gouvernement et le corps diplomatique. La déception et les regards sombres s'harmonisent avec la vision nocturne :

« [...] Ces malheureux refaisaient dans un morne silence le chemin qu'ils avaient, dans la matinée, fait si gaiement en chantant ; ils avaient vu le bateau sauveur. $[\ldots]$

Longtemps nous marchâmes sous le ·regard de ces soldats, obsédante vision dont le souvenir fait mal.

Mais la nuit était venue » (BOPPE 1917 : 131-132).

Le bateau Città-di-Bari arrive. Ses phares éclairent la réalité de la tragédie humaine. La lumière de ses phares incarne la culmination dramatique ; elle traduit l'échec d'un espoir vital du salut promis : « tremblants de fièvre, les réfugiés, vieillards, femmes et enfants » attendent :

«Subitement dans cette pluie sinistre, des lumières ${ }^{16}$ pointent de tous côtés. Assez près de nous sur la gauche, les feux d'un bateau ; de la terre on échangeait avec lui des signaux lumineux; nous étions donc enfin à Saint-Jean de Médua dont nous ne distinguions pas encore les quelques maisons. Mais les lumières qui nous entouraient nous indiquaient bien que nous nous trouvions sur cette plage funeste ; leur faible éclat provenait de ces foyers autour desquels se

${ }^{16}$ C'est nous qui soulignons. 
serraient, tremblants de fièvre, les réfugiés, vieillards, femmes et enfants, qui depuis des semaines attendaient, sans abri, un bateau chaque jour promis » (BOPPE 1917 : 132-133).

Le témoignage de guerre en tant que genre littéraire partage d'autres traits distinctifs avec les genres dramatiques : la parole y est spontanée, non-préparée au préalable ; ainsi, notre analyse confirme le propos théorique de Jeanelle qui affirme l'existence d' « une scénographie du témoignage » (2004 : 88).

Quant à la scénographie du témoignage analysé, on pourrait évoquer la description du décor de ce théâtre historique qui se déroule devant les yeux du témoin durant l'embarquement. La scénographie de l'intérieur du bateau est réaliste : y figurent les télégrammes, le verre de whisky, la tasse de thé... Les indications sur la lumière font écho à l'ambiance sombre de ce « spectacle inoubliable de détresse » du bateau qui est en train de partir de Médua (Albanie) vers Corfou (Grèce). Et c'est sur l'arrière-plan de ce décor qu'apparaît un personnage historique, monsieur Pašić17 ${ }^{17}$

«Cet homme, si maître de soi, que les vicissitudes les plus diverses ont toujours trouvé ferme et droit, ne peut dominer l'émotion qui l'étreint. Il va quitter la terre serbe, emmener à l'étranger le Gouvernement, l'armée... Ses souffrances morales donnent à son masque sévère, à sa longue barbe blanche, un aspect tragique. Un instant, nous restons silencieux devant cette douleur ; mais notre entrée a fait sortir le président du Conseil de son rêve ; d'une voix éteinte, il dit ses tristesses, son angoisse devant la gravité du moment, ses appréhensions pour l'avenir : «C'est l'image de la Serbie qui va s'embarquer», répète-t-il ; mais peu à peu les ministres serbes, abattus, déprimés, désemparés, viennent se grouper autour de M. Pachitch comme s'ils cherchaient un appui, un réconfort » (BOPPE 1917 : 135-136).

Comment est présenté ce personnage du drame historique d'un peuple? S'agit-il d'une nouvelle forme d'héroïsme littéraire qui conteste les modalités précédentes, romantique ou réaliste ? Il s'agit ici du changement de paradigme de la représentation de la guerre - pensons notamment à la scène de la bataille de Waterloo de Stendhal dans son œuvre La Chartreuse de Parme (1839). Nous pouvons constater que Cru et Stendhal à la fois, mais de façon différente, insistent sur le fait suivant : « démonter de trop faciles certitudes ». L'arme de Stendhal, aux dires de Mongin dans son article « Waterloo ou la pluralité des interprétations » est l'ironie : "Après Voltaire et Montesquieu, qui se servaient de Hurons et de Persans, mais en dissimulant mieux son ironie, Stendhal exploite la présence d'un naïf dans des circonstances connues et supposées bien comprises pour démonter de trop faciles certitudes » (MONGIN : 2010). Cependant, la dissimulation des idées reçues sur la guerre effectuée par les

${ }^{17}$ Nikola Pašić était le premier président du conseil du royaume des Serbes, des Croates et des Slovènes, le royaume a été proclamé le $1^{\text {er }}$ décembre 1918. 
témoins n'est possible qu'à travers la description réaliste des faits ; elle est conditionnée par la présence attestée aux événements évoqués. Le changement de paradigme de la représentation de la guerre est substantiel : si avec Stendhal on assiste à la fin du roman de guerre, à sa dérision, avec Boppe sommesnous à la découverte d'un surgissement, celui d'un nouveau genre? Si l'ironie stendhalienne met en cause les fondements de l'héroïsme romanesque dans le roman traditionnel, Boppe met en scène une nouvelle forme d'héroïsme littéraire - sans voix, le héros est présent ; par l'entremise de son rôle sur la scène historique, le héros est auto-suffisant. Sa parole est redondante - c'est au témoin, par son «être au monde », par son « être là » de transporter cet univers à la fois historique et littéraire. Par conséquent, deux possibilités, ou mieux encore, deux procédés de réception s'offrent aux lecteurs : si l'identification avec l'antihéros de Stendhal est ironique, l'identification avec la situation évoquée par Boppe est mimétique. Le mimétisme devient ainsi la condition sine qua non du genre testimonial.

Factualité ou émotion, histoire ou littérature ? Le récit de Boppe amalgame les deux pôles, ce qui permet de changer la perspective : en partant de celle de l'historien soucieux de dater les passages de la traversée et de les décrire avec une précision exacte, le regard de Boppe se focalise par la suite sur les hommes, sur leur destin, sur leurs conditions, sur leurs visions... De cette manière, sa posture change - il prend la plume de l'écrivain.

Sa parole relève à la fois de l'Histoire et de la littérature et transcende les deux champs du savoir. Parole réaliste contre les représentations légendaires et mythiques, parole subjective qui donne accès à hic et nunc de ce théâtre d'Histoire - n'est-ce pas la double dimension qui fait la vraie valeur du genre testimonial ? Au-delà de sa portée historique et éthique, qui consiste à dire la vérité de cet événement tragique de l'histoire nationale, le lecteur se retrouve face à une vérité encore plus profonde : c'est celle de la vérité individuelle, humaine-littéraire.

\section{Bibliographie}

CRU 2002 : CRU, Jean Norton. Du témoignage. Internet : Éditions de l'Aaargh, 2002.

HARTOG 2017 : HARTOG, François. « La présence du témoin ». L'Homme $n^{\circ} 223-224$ (2017) : 169-184.

HARTOG 2007 : HARTOG, François. Évidence de l'histoire. Ce que voient les historiens. Paris : Gallimard, 2007.

JEANELLE 2004 : JEANELLE, Jean-Louis. « Pour une histoire du genre testimonial ». Littérature $n^{\circ} 135$ (2004) : 87-117. 
MONGIN 2010 : MONGIN, Philippe. «Waterloo ou la pluralité des interprétations ». Littérature vol. 165, $\mathrm{n}^{\circ} 1$ (2012): 84-113.

PAVLOVIĆ 2006 : PAVLOVIĆ, Vojislav. « La France et le programme yougoslave du gouvernement serbe. » Balcanica, vol. 37. (2006) : 171193.

SRETENOVIĆ 2012 : SRETENOVIĆ, Stanislav. « Le monument à la France à Belgrade. La mémoire de la Grande Guerre au service de l'action politique et diplomatique ». Vingtième Siècle, Revue d'histoire ${ }^{\circ} 115$ $(2012 / 3): 31-44$.

\section{Sources}

BOPPE 1917 : BOPPE, Auguste. À la suite du gouvernement Serbe, de Nich à Corfou, 20 octobre 1915-19 janvier 1916. Paris : Éditions Bossard, 1917.

PAVLOVIĆ 1988 : PAVLOVIĆ, Mihailo. Témoignages français sur les Serbes et la Serbie 1912-1918, choix de textes, notes de présentation, traduction et commentaires par Mihailo Pavlović, (Francuzi o Srbima i Srbiji : 1912-1918, izbor, uvodni tekstovi, prevod i komentari Mihailo Pavlović), édition bilingue. Belgrade : Narodna knjiga, 1988.

Présentation Internet de l'Ambassade de France en Serbie, sous le volet Actualités de la coopération > Mémoire franco-serbe : <https:// rs.ambafrance.org/-Ambassade-> dernière consultation le 3/11/2019.

Драгана С. Цветковић

НА РАСКРШЋУ ИЗМЕЪУ ИСТОРИЈЕ И КЬИЖЕВНОСТИ: АНАЛИЗА РАТНОГ СВЕДОЧЕЬА ЗА СРПСКОМ ВЛАДОМ ОД НИША ДО КРФА: ОД 20. ОКТОБРА 1915. ДО 19. ЈАНУАРА 1916. ОГИСТА БОПА

Француско-српски односи су део дуге традиције која почиње током средњег века. Ојачавши током деветнаестог века, француско-српско пријатељство се још више учвршћава кроз „пријатељство у оружју” током Великог рата. Многи документи и лична сећања нам дају увид о овој реалности, међу којима је и ратно сведочење француског дипломате Огиста Бопа За српском владом од Ниша до Крфа: од 20. октобра 1915. до 19. јануара 1916. чији делови су предмет наше анализе. Следеће перспективе оријентишу наше истраживање: имајући у виду чињеницу да књижевност сведочења књижевни жанр који се налази на раскршћу дисциплина и знања - заузима посебан статус у савременој књижевној мисли у Француској, ми ћемо испитивати књижевне, историјске и етичке домете тог „чина сведочења”. 
Питање је двоструко; етичко и методолошко, најпре, да се искаже истина онога што је преживљено у име жртава, и књижевно и естетско, зато што се та реалност приказује кроз субјективну призму нарације. Наш методолошки приступ се, дакле, заснива на теоријској основи француског критичара Жан-Луја Жанела који издваја поменуте компоненте ратног сведочења као књижевног жанра: етичку, методолошку и естетску. Резултати анализе показују да ратно сведочење превазилази дијалектику између фактуелно-историјског и књижевно-естетског домена. Управо та дупла димензија приказује праву вредност овог жанра - спојити истину историјског догађаја са истином искуства тог догађаја.

Кључне речи: књижевни жанр, ратно сведочење, историја, Велики рат, француско-српски односи 